The Astrophysical Journal, 450:L11-L15, 1995 September 1

(C) 1995. The American Astronomical Society. All rights reserved. Printed in U.S.A.

\title{
THE TYPE Ic SUPERNOVA 1994I IN M51: DETECTION OF HELIUM AND SPECTRAL EVOLUTION
}

\author{
Alexei V. Filippenko, ${ }^{1,2}$ Aaron J. Barth, ${ }^{1}$ Thomas Matheson, ${ }^{1}$ Lee Armus,${ }^{3}$ Michael Brown, ${ }^{1,4}$ Brian R. Espey, ${ }^{5}$ \\ Xiao-Ming Fan, ${ }^{6,7}$ Robert W. Goodrich, ${ }^{8,9}$ Luis C. Ho, ${ }^{1}$ Vesa T. JunkKarinen, ${ }^{6}$ David C. Koo, ${ }^{8}$ \\ Matthew D. Lehnert, ${ }^{10}$ André R. Martel, ${ }^{8}$ Joseph M. Mazzarella, ${ }^{11}$ Joseph S. Miller,${ }^{8}$ \\ Graeme H. Smith, ${ }^{8}$ David Tytler, ${ }^{6}$ And Gregory D. Wirth ${ }^{8}$ \\ Received 1995 May 19; accepted 1995 June 14
}

\begin{abstract}
We present a series of spectra of SN 1994I in M51, starting 1 week prior to maximum brightness. The nebular phase began about 2 months after the explosion; together with the rapid decline of the optical light, this suggests that the ejected mass was small. Although lines of $\mathrm{He}$ I in the optical region are weak or absent, consistent with the Type Ic classification, we detect strong He I $\lambda 10830$ absorption during the first month past maximum. Thus, if SN 1994I is a typical Type Ic supernova, the atmospheres of these objects cannot be completely devoid of helium. The emission-line widths are smaller than predicted by the model of Nomoto and coworkers, in which the iron core of a low-mass carbon-oxygen star collapses. They are, however, larger than in Type Ib supernovae.

Subject headings: binaries: close — stars: evolution — stars: mass loss — supernovae: general — supernovae: individual (SN 1994I, SN 1987M, SN 1984L)
\end{abstract}

\section{INTRODUCTION}

There are three main subclasses of hydrogen-deficient (Type I) supernovae (SNs) defined by their optical spectra within $\sim 1$ month past maximum brightness (see, e.g., Harkness \& Wheeler 1990; Filippenko 1991a). SNs Ia exhibit a deep absorption trough near $6150 \AA$, thought to be due to blueshifted Si II $\lambda 6355$. This feature is absent in SNs Ib, which instead have prominent lines of $\mathrm{He}$ I, while SNs Ic show neither Si II nor He I. Many months past maximum brightness, the nebular spectra of SNs Ia are riddled with blends of iron-group elements, whereas those of $\mathrm{SNs} \mathrm{Ib}$ and Ic are dominated by a few strong emission lines of intermediate-mass elements $(\mathrm{O}, \mathrm{Mg}, \mathrm{Ca})$.

SNs Ia result from thermonuclear runaway in C-O white dwarfs, probably close to the Chandrasekhar limit; see Woosley \& Weaver (1986) for a review. Although white dwarfs might also be responsible for SNs Ib and Ic, these objects are now generally believed to be massive stars undergoing core collapse after having lost their outer envelope of gas (e.g., Branch, Nomoto, \& Filippenko 1991; Wheeler \& Harkness 1991); hence, they are fundamentally related to hydrogen-rich (Type II) SNs. The origins of the observed differences between SNs Ib and Ic, as well as the exact nature of their progenitor

\footnotetext{
${ }^{1}$ Department of Astronomy, University of California, Berkeley, CA 94720 ${ }^{2}$ Center for Particle Astrophysics, University of California, Berkeley, CA 94720.

${ }^{3}$ Downs Laboratory of Physics, 320-47 Caltech, Pasadena, CA 91125.

${ }^{4}$ Current address: Lunar and Planetary Laboratory, University of Arizona, Tucson, AZ 85721.

${ }^{5}$ Center for Astrophysical Sciences, Johns Hopkins University, Baltimore, MD 21218.

${ }^{6}$ Center for Astrophysics and Space Sciences, University of California, San Diego, CA 92093.

${ }^{7}$ Current address: Department of Astronomy, Columbia University, New York, NY 10027.

${ }^{8}$ Lick Observatory, University of California, Santa Cruz, CA 95064.

${ }_{9}^{9}$ Current address: Space Telescope Science Institute, 3700 San Martin Drive, Baltimore, MD 21218.

${ }^{10}$ Institute of Geophysics and Planetary Physics, Lawrence Livermore National Laboratory, P.O. Box 808, L-413, Livermore, CA 94550.

${ }^{11}$ Infrared Processing and Analysis Center, MS 100-22, California Institute of Technology, Pasadena, CA 91125.
}

stars and their presupernova evolution, are still controversial. Wheeler et al. (1987), for example, argue that helium is physically absent from SNs Ic, whereas Hachisu et al. (1991; see also Nomoto, Filippenko, \& Shigeyama 1990) suggest that helium is present but not excited to the appropriate energy levels. Progress in this field rests largely on the availability of new observations spanning a wide wavelength range, from early to late epochs.

An excellent opportunity to improve the database was provided by SN 1994I in the bright, nearby galaxy M51 (NGC 5194). This object, independently discovered by at least four groups during the interval 1994 April 2.17-2.66 UT (Puckett et al. 1994), was eventually classified as a SN Ic (Schmidt et al. 1994; Clocchiatti et al. 1994) after some initial confusion. Here we show a series of optical and near-infrared (near-IR) spectra of SN 1994I; preliminary results were given by Filippenko et al. (1994a). Our optical light curves of SN 1994I will be published in a subsequent paper (Richmond et al. 1995).

\section{OBSERVATIONS AND REDUCTIONS}

Spectra of SN 1994I were taken over a 5 month interval starting from 1994 April 2.30 UT, just a few hours after discovery. For all but the first spectrum, which was obtained with the $5 \mathrm{~m}$ Hale reflector at Palomar Observatory, we used the Kast double spectrograph (Miller \& Stone 1993) at the Cassegrain focus of the Shane $3 \mathrm{~m}$ reflector at Lick Observatory. The long, narrow (in most cases $2^{\prime \prime}$ ) slit was generally oriented along the parallactic angle to minimize differential light losses produced by atmospheric dispersion. The total spectral coverage (up to 3100-11400 $\AA$ ) and resolution (typically $5-15 \AA$ ) varied from night to night, depending on the other observing programs.

One-dimensional spectra were extracted from the CCD data in the normal manner; the VISTA set of routines was used in all but one case. Since SN 1994I lies $\sim 19^{\prime \prime}$ from the nucleus of $\mathrm{M} 51$, in a very complicated region, the sky background was chosen as close as possible to the star. However, residual galaxy contamination is present in some spectra, especially at late times. We used our own software for flux calibration, 


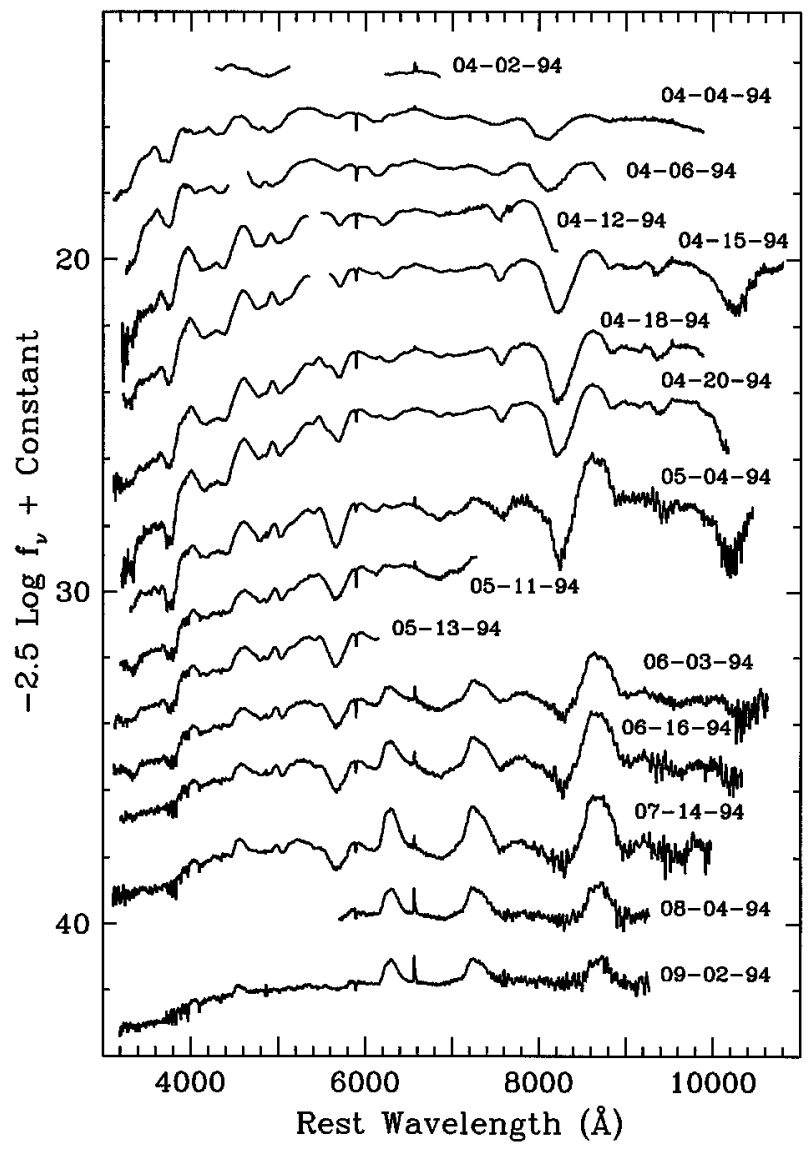

FIG. 1.-Montage of spectra of SN 1994I. The ordinate units are magnitudes. UT dates are indicated; V maximum was on April 9. The May 13 spectrum is an average of data obtained on May 12 and 13. Telluric absorption lines were removed, but the spectra were not dereddened. In some spectra residual CCD fringing redward of $\sim 7400 \AA$ is visible. The late-time spectra are significantly contaminated by gas and early-type stars in the host galaxy; note the blue continuum, as well as the Balmer absorption and emission lines. Blueshifted He I $\lambda 10830$ is prominent at early times, and the transition to the nebular phase is rapid.

wavelength calibration, and removal of telluric features; see Filippenko, Matheson, \& Ho (1993).

Of particular importance is the spectral region beyond 9000 $\AA$, since we were hoping to detect or set limits on the He I $\lambda 10830$ line. A dichroic beam splitter was used to transmit light having $\lambda \gtrsim 5400 \AA$ to the red camera, although the transmission is nonzero down to $\sim 5200 \AA$. (On April 6 the dichroic transmitted $\lambda \gtrsim 4500 \AA$, and on May 12 and 13 only the blue camera was used.) Thus, in general the spectra have no second-order contamination up to $10400 \AA$, are somewhat less reliable in the range 10400-10800 $\AA$, and have been truncated beyond $10800 \AA$. The June 3 and July 14 spectra were obtained with an OG550 order-blocking filter, making second-order light absent shortward of $10800 \AA$.

\section{RESULTS}

A montage showing most of our spectra is illustrated in Figure 1, along with the corresponding UT dates. For reference, visual maximum (12.9 mag) was on 1994 April 9 ( $t \equiv 0$; Yokoo et al. 1994; Richmond et al. 1995). The spectra have not been corrected for interstellar extinction, which is likely to be $A_{V} \approx 1.4 \mathrm{mag}$; see Iwamoto et al. (1994; $A_{V}$ determined from an overall fit to the light curve), Baron et al. (1995; spectral fits), and Ho \& Filippenko (1995; measurements of $\mathrm{Na}$ I $\mathrm{D}$ absorption, most of which is in the host galaxy M51). The redshift $\left(c z \approx 500 \mathrm{~km} \mathrm{~s}^{-1}\right)$, as measured from the narrow $\mathrm{H} \alpha$ (or in some cases $\mathrm{H} \beta$ ) emission line produced by superposed $\mathrm{H}$ II regions, has been removed.

The first spectrum (April 2) reveals an essentially flat continuum with a few very broad, low-amplitude undulations. Over the next few nights the spectrum evolves rapidly (see also Sasaki et al. 1994), with Ca II H $+\mathrm{K}$ and Ca II near-IR triplet absorption being the strongest features. The blueshift of the Ca II near-IR absorption minimum quickly decreases, presumably because we see progressively deeper layers of homologously expanding gas. The $\mathrm{Ca}$ II and other absorption lines gradually grow stronger as the continuum reddens; specifically, the blends of $\mathrm{Fe}$ II lines around 4200 and $4700 \AA$ s soon become prominent, as does $\mathrm{O}$ i $\lambda 7774$. Nebular emission lines, especially [O I] $\lambda \lambda 6300,6364$ and [Ca II] $\lambda \lambda 7291,7324$, are strong in the late-time spectra.

We agree with Sasaki et al. (1994) and Wheeler et al. (1994), who classified SN 1994I as Type Ic. $\mathrm{H} \alpha$ is weak or absent, although there is a local maximum at $\sim 6560 \AA$ consistent with that seen in some other SNs Ic (Filippenko 1992). The dip at $\sim 6200 \AA$ might be Si II $\lambda 6355$, but this feature is much more subtle than in classical SNs Ia. Finally, He I lines are not obvious in the optical region. ${ }^{12}$

There may be evidence for weak $\mathrm{He}$ I $\lambda 5876$ absorption. A shallow minimum is visible (Fig. 1) at $\sim 5560 \AA$ on April 4 and 18, and to a lesser extent on April 6 and 20, possibly corresponding to $\mathrm{He}$ I $\lambda 5876$ at $v=-16,600 \mathrm{~km} \mathrm{~s}^{-1}$. Detailed comparisons of spectra by A. Clocchiatti (1995, private communication) support this interpretation for SN 1994I as well as for the Type Ic SN 1987M (Filippenko, Porter, \& Sargent 1990). Alternatively, one can argue that the line at $\sim 5680 \AA$ is contaminated by He I, being stronger in SN 1994I than in SN 1987M. This can be seen in Figure 2; the top panel compares SN 1994I (1994 April 15; $t=6$ days) with SN 1987M (1987 September 28; $t \approx 7$ days; Filippenko et al. 1990) and SN Ib 1984L (1984 September 3; $t \approx 2$ days; Harkness et al. 1987), while the bottom panel compares SN 1994I (1994 May 4; $t=25$ days) with SN 1987 M (1987 October $20 ; t \approx 29$ days) and SN 1984L (1984 September 23; $t \approx 22$ days). Of course, Na I D might simply be stronger in SN 1994I than in SN 1987M at all times, in which case no contribution from He I $\lambda 5876$ is necessary in the $5680 \AA$ feature.

Direct evidence for the physical presence of helium in SN 1994I is provided by the prominent $\mathrm{He}$ I $\lambda 10830$ absorption, as shown in Figures 1 and 2. This line is expected to be the strongest $\mathrm{He}$ I transition over the observed wavelength range (Swartz et al. 1993b); indeed, it should be sensitive to very small amounts of helium (Wheeler, Swartz, \& Harkness 1993; Baron et al. 1995). The observed feature cannot be an artifact produced by second-order contamination $(\S 2)$ because its minimum is at $\sim 10250 \AA\left(v \approx-16,500 \mathrm{~km} \mathrm{~s}^{-1}\right)$ and the blue wing clearly extends all the way to $\sim 9800 \AA(v \approx-29,900 \mathrm{~km}$ $\mathrm{s}^{-1}$ ). Besides He I, the most plausible identification is $\mathrm{Mg}$ II $\lambda 10930$, which is strong in some synthetic spectra of SNs Ia (Baron \& Nugent 1995, private communication). However, this line does not appear in the He star models of Swartz et al.

12 The spectrum of April 15.69 in Sasaki et al. (1994) contains a very strong absorption line at $4800 \AA$ which they attribute to He I $\lambda 4471$ (but they probably meant He I 15015$)$. Comparison with Fig. 1 suggests that it is an artifact. 


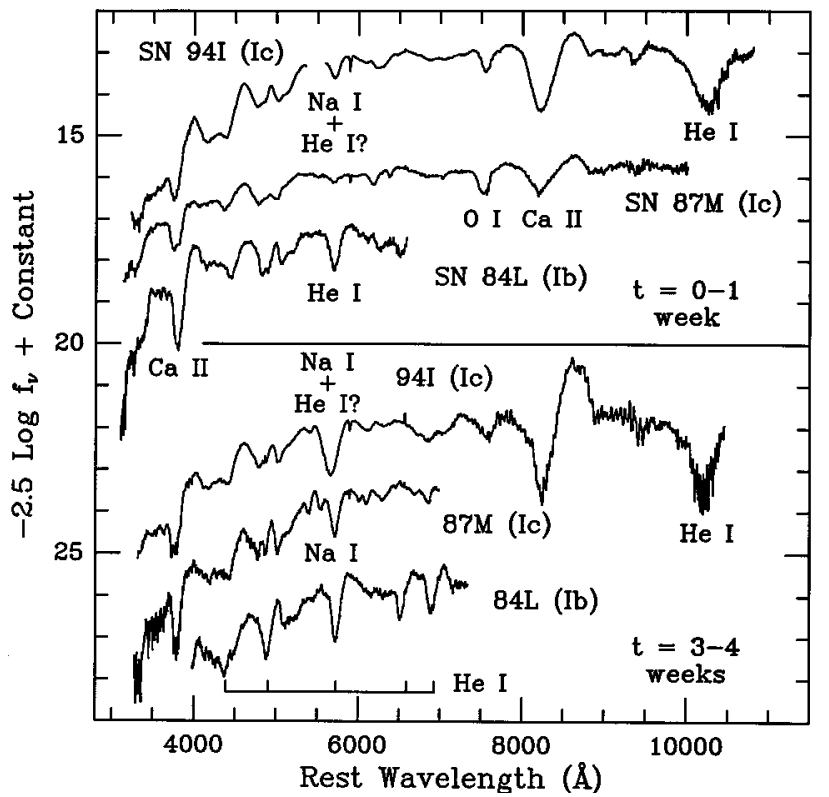

FIG. 2.- Spectra of SNs Ib and Ic obtained 0-1 week (top) and 3-4 weeks (bottom) past maximum $V$ brightness, compared with those of SN 1994I. He I $\lambda 5876$ is absent in SN Ic 1987M, strong in SN Ib 1984L, and possibly present (but weak, and contaminated by Na I D) in SN 1994I. Based on the optical region alone, the classification of SN 1994I is clearly Ic, but the strong He $\lambda 10830$ line reveals the presence of helium. SN 1994I appears redder than the other two SNs, largely because of its high extinction $\left(A_{V} \approx 1.4 \mathrm{mag}\right)$.

(1993b), and the observed absorption minimum would correspond to an unusually high velocity of $-19,200 \mathrm{~km} \mathrm{~s}^{-1}$. If, on the other hand, the feature is identified with He I, then its velocity $\left(-16,500 \mathrm{~km} \mathrm{~s}^{-1}\right)$ is in excellent agreement with that of the possible He I $\lambda 5876$ line at $5560 \AA(v \approx-16,600 \mathrm{~km}$ $\mathrm{s}^{-1}$ ), as pointed out by Clocchiatti (1995, private communication). Hence, we feel that the identification of He I $\lambda 10830$ in SN 1994I is secure.

The He I $\lambda 10830$ absorption line in SN 1994I appears on April 15, 16 (not shown in Fig. 1), and 20 and May 4; the extreme blue wing might also be present in the spectrum of April 18. A corresponding P Cygni emission component seems weak or absent, but note that it might have been artificially suppressed; the 10400-10800 $\AA$ region was susceptible to second-order contamination, and the derived flux of SN 1994I would therefore be too low if calibrated against a bluer standard star. The absorption line is much weaker, but probably still present, in the June 3 and 16 spectra.

This represents the first undeniable detection of $\mathrm{He} \mathrm{I}$ in a SN Ic, although no other SNs Ic have been observed at $\lambda \gtrsim 10000 \AA$. Wheeler et al. (1994) show strong He I $\lambda 10830$ absorption in a near-IR spectrum of SN $1990 \mathrm{~W}$, which was originally classified as Type Ic, but they argue that weak He I absorption is visible in optical spectra and that the object's light curve is more consistent with those of SNs Ib; hence, SN 1990W might actually be a relatively helium-poor SN Ib. Several other SNs Ic may exhibit He I $\lambda 5876$ (e.g., SN 1988L [Filippenko 1988]; SN 1990U [Gómez \& López 1994]), but significant uncertainties in the strength of $\mathrm{Na}$ I $\mathrm{D}$ absorption and in the phase at the time of observation complicate the interpretation (Filippenko 1991b). Although much more work is needed in this area, there may be a wide range of $\mathrm{He} \mathrm{I}$ strengths; if so, classical SNs Ib and Ic are just the extrema of the distribution. Additional near-IR spectra are needed to

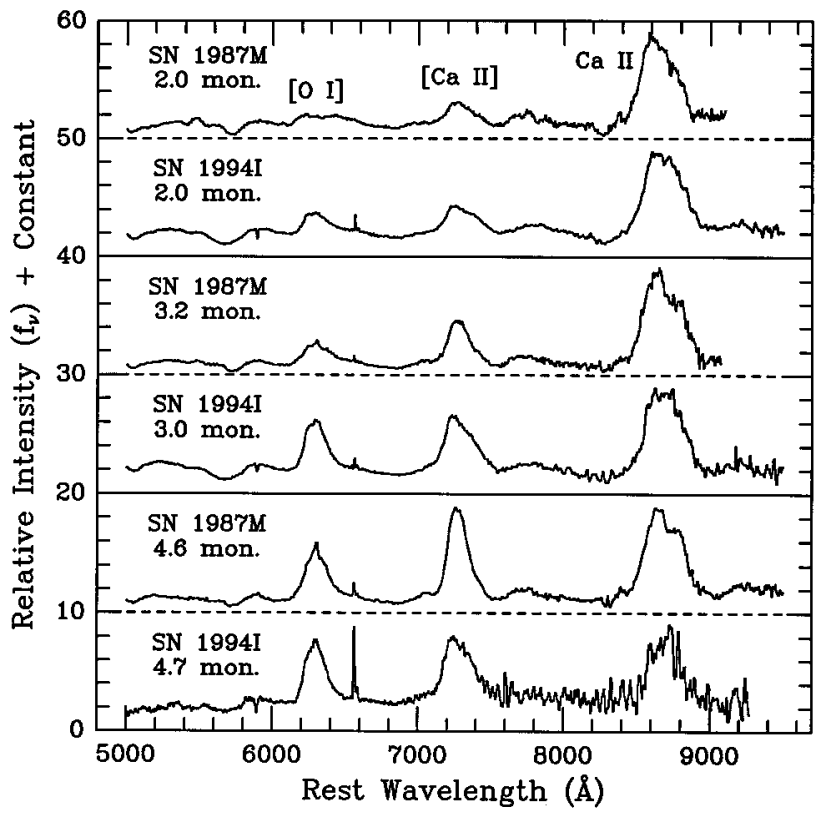

FIG. 3.-Comparison of the spectra of the Type Ic SNs 1994I and 1987M in the nebular phase. The spectrum of SN 1994I at $t=2.0$ months past $V$ maximum is the average of data obtained on June 3 and 16. To emphasize the emission lines, the bright stellar continuum has been partially removed from the spectrum of SN 1994I at $t=4.7$ months. The spectra have been scaled so that the strength of $\mathrm{Ca}$ II is similar in all cases. Note the differences in line width and the $[\mathrm{O} \mathrm{I}] /[\mathrm{Ca}$ II] intensity ratio. The Ca II line in SN $1987 \mathrm{M}$ is probably contaminated by [C I] $\lambda 8727$ emission.

determine whether any SNs Ic show no evidence of He I $\lambda 10830$ absorption.

SN 1994I made a rapid transition to the nebular phase; prominent [O I] $\lambda \lambda 6300,6364$ and [Ca II] $\lambda \lambda 7291,7324$ are visible in the spectrum of June 3 (Fig. 1), less than 2 months past maximum brightness. This is very similar to the behavior of Type Ic SN 1987M (Filippenko et al. 1990), and it suggests that the mass of the ejecta is small. The optical light curves support this conjecture; in both cases (SN 1987M [Filippenko et al. 1990]; SN 1994I [Richmond et al. 1995]) they fall more rapidly than those of SNs Ia (e.g., Doggett \& Branch 1985) and SNs Ib (e.g., Schlegel \& Kirshner 1989).

A detailed comparison of the late-time spectra of SNs $1987 \mathrm{M}$ and 1994I is given in Figure 3 for three phases. Note that the $[\mathrm{O} \mathrm{I}] /[\mathrm{Ca} \mathrm{II}]$ intensity ratio is larger in SN 1994I than in $\mathrm{SN} 1987 \mathrm{M}$ at all times, suggesting a difference in their oxygen content relative to calcium (rather than a difference in ionization state)..$^{13}$ At each phase, the [Ca II] line is considerably broader in SN 1994I than in SN 1987M; for example, at $t \approx 4.6$ months, the FWHM is $9200 \mathrm{~km} \mathrm{~s}^{-1}$ for SN 1994I, compared with only $6200 \mathrm{~km} \mathrm{~s}^{-1}$ for SN 1987M. Perhaps SN 1994I had a higher explosion energy and/or a smaller ejected mass than did SN 1987M. The [O I] profile in SN 1994I, on the other hand, is only slightly broader than in SN 1987M: at $t \approx 4.6$ months, we measure FWHM $=7700$ and $7500 \mathrm{~km} \mathrm{~s}^{-1}$, respectively. The Ca II near-IR profile of SN 1994I appears narrower than that of SN $1987 \mathrm{M}$ at $t \approx 3.1$ and 4.6 months, but this may be due to the presence of blended [C I] $\lambda 8727$ emission in the latter. Note that the widths of the [O $\mathrm{I}]$ and

13 Curiously, at early times the O I/Ca II absorption ratio is smaller in SN 1994I than in SN 1987M (Fig. 2, top). This may, however, reflect differences in the "primordial" calcium content, since the early photospheric Ca II absorption should not be dominated by freshly synthesized atoms. 
[Ca II] lines in late-time spectra of SNs Ib (FWHM $=4500 \pm$ $500 \mathrm{~km} \mathrm{~s}^{-1}$; Schlegel \& Kirshner 1989) are significantly smaller than those in the Type Ic SN 1987M and SN 1994I.

\section{DISCUSSION}

The general consensus is that the progenitors of SNs Ib and Ic are massive stars undergoing core collapse after having shed their outer envelope of gas (e.g., Filippenko et al. 1990 and references therein). A direct link between SNs II and SNs Ib/Ic was provided by SN 1987K (Filippenko 1988) and SN 1993J (Filippenko et al. 1993; Swartz et al. 1993a; Filippenko, Matheson, \& Barth 1994b): at early times these two objects displayed prominent hydrogen lines, but $\sim 6$ months later their spectra were nearly indistinguishable from those of $\mathrm{SNs} \mathrm{Ib} / \mathrm{Ic}$.

There is, however, a diversity of specific models for corecollapse SNs $\mathrm{Ib} / \mathrm{Ic}$. One possibility is that the progenitors are very massive stars that lost their entire $\mathrm{H}$ (SNs Ib) or $\mathrm{H}$ plus $\mathrm{He}$ (SNs Ic) layers primarily through winds, as in the case of Wolf-Rayet stars (e.g., Woosley, Langer, \& Weaver 1993; Swartz et al. 1993b and references therein). Alternatively, the mass loss could have occurred primarily as a result of transfer to a bound companion (Nomoto et al. 1994). Hybrid models are also plausible, with the $\mathrm{H}$ being largely transferred to a companion star and the He being depleted by efficient massdependent mass loss (Woosley, Langer, \& Weaver 1995); the classification of the resulting SN (Ib or Ic) depends on the amount of remaining He. Hachisu et al. (1991) suggested that the He envelope might be present even in SNs Ic, but this is now considered unlikely, since strong $\mathrm{He}$ I lines should then be visible (Swartz et al. 1993b).

Two different groups have successfully modeled the observed light curve of SN 1994I in an attempt to determine the nature of the progenitor. Nomoto et al. (1994; see also Iwamoto et al. 1994) suggest that the object was a $2.2 M_{\odot} \mathrm{C}-\mathrm{O}$ core formed as a consequence of two stages of mass transfer in a binary system; during the second stage, He was lost to the close companion (most likely an $\mathrm{O}-\mathrm{Ne}-\mathrm{Mg}$ white dwarf). The Fe core of the C-O star collapsed, and the ejecta are less massive than those of a completely disrupted Chandrasekharmass white dwarf; thus, the light curve declined more rapidly than those of SNs Ia. Woosley et al. (1995), on the other hand, invoke only the first stage of mass transfer, during which the $\mathrm{H}$ envelope is lost to the companion. Depending on the initial mass of the star, the resulting He star has a mass in the range 4-20 $M_{\odot}$, but subsequent mass loss through winds is very efficient and makes the final mass of the $\mathrm{C}-\mathrm{O}$ star converge to the narrow range $2.26-3.55 M_{\odot}$ in all cases.

The two groups predict rather different amounts of $\mathrm{He}$ in the progenitor stars immediately prior to the explosion. In the 2.2 $M_{\odot}$ model of Nomoto et al. (1994), the C + O envelope has a mass of $0.14 M_{\odot}$ and a helium mass fraction of 0.09 ; thus, the total He mass is only $\sim 0.01 M_{\odot}$, although somewhat more $\mathrm{He}$ would remain if the second stage of mass transfer were incomplete. The progenitors modeled by Woosley et al. (1995), by contrast, typically have $0.1-0.3 M_{\odot}$ of $\mathrm{He}$ in the envelope. Thus, our data can provide a direct test of these predictions, which differ by roughly an order of magnitude.

Unfortunately, without doing a thorough analysis we are unable to estimate the He mass implied by the He I $\lambda 10830$ line. To get any He absorption at all, enormous departures from local thermodynamic equilibrium (LTE) are necessary (Harkness et al. 1987; Lucy 1991; Swartz et al. 1993b). The upper levels of He are populated by collisional excitations with primary (or secondary) nonthermal electrons, which come from atoms that have interacted with the $\gamma$-rays produced by radioactive decay of ${ }^{56} \mathrm{Ni}$ and ${ }^{56} \mathrm{Co}$. Baron et al. (1995) did not find any direct evidence for $\mathrm{He}$ (upper limit of $\sim 0.1 M_{\odot}$ ) in a preliminary non-LTE analysis of optical spectra of SN 1994I. A more complete treatment which includes the $\mathrm{He}$ I $\lambda 10830$ line is in progress.

Nomoto et al. (1994) make a specific prediction about the late-time spectrum of SN 1994I: emission lines of Ca should exhibit velocities of up to $\sim 10,000 \mathrm{~km} \mathrm{~s}^{-1}$, and the $\mathrm{O}$ lines would be even broader, since $\mathrm{O}$ is concentrated in the outermost gas layers (Iwamoto et al. 1994). However, this is not the case (Fig. 3 and $\S 3$ ); although it consists of the more closely spaced doublet, the [Ca II] line is broader than [O I], and the most rapidly moving gas (as measured by the half-width near zero intensity) is less than $7000 \mathrm{~km} \mathrm{~s}^{-1}$ for [O I]. In SN 1987M, on the other hand, [O I] might be slightly broader than [Ca II], but the highest observed velocities are still substantially below the predicted values.

In $\S 3$ we suggested that SN 1994I has a higher O content relative to $\mathrm{Ca}$ than does SN 1987M. If interpreted in the context of the Nomoto et al. (1994) scenario, this might imply that the progenitor of SN 1994I was somewhat more massive than that of SN 1987M. Specifically, the $1.8 M_{\odot}$ model of Iwamoto et al. (1994) produces an O/Ca mass ratio of 44, while the $2.1 M_{\odot}$ model produces a ratio nearly 4 times larger. It remains to be seen whether other predictions of these models can be reconciled with the observations.

Detailed analysis of the spectra of SN 1994I, together with additional theoretical calculations, should lead to a better understanding of the progenitors and explosion mechanisms of SNs Ic. A comparison between the predicted and observed masses of helium will be the most important initial step, but the light curves and expansion velocities also provide valuable constraints on the models.

This work was supported by grants from NSF, most recently AST-9417213; from NASA, especially GO-2563.01-87A from STScI (operated by AURA, Inc., under NASA contract NAS5-26555); and from the DOE (contract W-7405-ENG-48). We thank the Lick staff for their assistance, and R. P. Harkness for the digital spectrum of SN 1984L (Fig. 2).

\section{REFERENCES}

Baron, E., Hauschildt, P. H., Branch, D., Kirshner, R. P., \& Filippenko, A. V. 1995, MNRAS, submitted

Branch, D., Nomoto, K., \& Filippenko, A. V. 1991, Comments Astrophys., 15, 221

Clocchiatti, A., Brotherton, M., Harkness, R. P., \& Wheeler, J. C. 1994, IAU Circ. 5972

Doggett, J., \& Branch, D. 1985, AJ, 90, 2303

Filippenko, A. V. 1988, AJ, 96, 1941

. 1991a, in Supernovae and Stellar Evolution, ed. A. Ray \& T. Velusamy

(Singapore: World Scientific), 34
Filippenko, A. V., 1991b, in SN 1987A and Other Supernovae, ed. I. J. Danziger \& K. Kjär (Garching: ESO), 343 . 1992, ApJ, 384, L37

Filippenko, A. V., Barth, A. J., Matheson, T., \& Ho, L. C. 1994a, BAAS, 26 , 1361

Filippenko, A. V., Matheson, T., \& Barth, A. J. 1994b, AJ, 108, 2220

Filippenko, A. V., Matheson, T., \& Ho, L. C. 1993, ApJ, 415, L103

Filippenko, A. V., Porter, A. C., \& Sargent, W. L. W. 1990, AJ, 100, 1575

Gómez, G., \& López, R. 1994, AJ, 108, 195

Hachisu, I., Matsuda, T., Nomoto, K., \& Shigeyama, T. 1991, ApJ, 368, L27 
Harkness, R. P., \& Wheeler, J. C. 1990, in Supernovae, ed. A. G. Petschek (New York: Springer), 1

Harkness, R. P., et al. 1987, ApJ, 317, 355

Ho, L. C., \& Filippenko, A. V. 1995, ApJ, 444, 165

Iwamoto, K., Nomoto, K., Höflich, P., Yamaoka, H., Kumagai, S., \& Shigeyama, T. 1994, ApJ, 437, L115

Lucy, L. B. 1991, ApJ, 383, 308

Miller, J. S., \& Stone, R. P. S. 1993, Lick Obs. Tech. Rep. 66

Nomoto, K., Filippenko, A. V., \& Shigeyama, T. 1990, A\&A, 240, L1

Nomoto, K., et al. 1994, Nature, 371, 227

Puckett, T., Armstrong, J., Johnson, W., Millar, D., Berry, R., \& Kushida, R. 1994, IAU Circ. 5961

Richmond, M. W., et al. 1995, AJ, submitted

Sasaki, M., Kosugi, G., Ishigaki, T., Maemura, H., Aoki, K., \& Ohtani, H. 1994, PASJ, 46, L187

Schlegel, E. M., \& Kirshner, R. P. 1989, AJ, 98, 577

Schmidt, B., Challis, P., Kirshner, R., Berland, P., \& Phillips, M. 1994, IAU Circ. 5966
Swartz, D. A., Clocchiatti, A., Benjamin, R., Lester, D. F., \& Wheeler, J. C. 1993a, Nature, 365, 232

Swartz, D. A., Filippenko, A. V., Nomoto, K., \& Wheeler, J. C. 1993b, ApJ, 411,313

Wheeler, J. C., \& Harkness, R. P. 1990, Rep. Prog. Phys., 53, 1467

Wheeler, J. C., Harkness, R. P., Barker, E. S., Cochran, A. L., \& Wills, D. 1987, ApJ, 313, L69

Wheeler, J. C., Swartz, D. A., \& Harkness, R. P. 1993, Phys. Rep., 227, 113

Wheeler, J. C., Harkness, R. P., Clocchiatti, A., Benetti, S., Brotherton, M. S. DePoy, D. L., \& Elias, J. 1994, ApJ, 436, L135

Woosley, S. E., Langer, N., \& Weaver, T. A. 1993, ApJ, 411, 823

. 1995, ApJ, 448, 315

Woosley, S. E., \& Weaver, T. A. 1986, ARA\&A, 24, 205

Yokoo, T., Arimoto, J., Matsumoto, K., Takahashi, A., \& Sadakane, K. 1994, PASJ, 46, L191 